\title{
A NOVEL APPROACH IN IDENTIFICATION OF BLOOD GROUP USING LASER TECHNOLOGY
}

\author{
Priyadharshini.R ${ }^{1}$, Ramya.S ${ }^{2}$, Kalaiyarasi.S ${ }^{3}$, Kalpana Devi.S $S^{4}$, SuthathiraVanitha. $N^{5}$ \\ ${ }^{I}$ B.E/EEE IInd year, Knowledge Institute of Technology, Tamilnadu, India \\ ${ }^{2}$ B.E/EEE IInd year, Knowledge Institute of Technology, Tamilnadu, India \\ ${ }^{3}$ B.E/EEE IInd year, Knowledge Institute of Technology, Tamilnadu, India \\ ${ }^{4}$ Associate professor/EEE, Knowledge Institute of Technology, Tamilnadu, India \\ ${ }^{5}$ Professor, Department of EEE, Knowledge Institute of Technology, Tamilnadu, India
}

\begin{abstract}
In this research paper, the proposed idea is to replace the manual work in clinical laboratories for identifying the blood group. Nowadays even though automation plays a vital role in all medical applications but still no device is available to determine the blood group automatically. Usually, the blood group analysis is made by the technicians in the laboratories. They are using the following methods for analysing the blood group 1.ABO forward typing 2.ABO reverse typing 3.Rhesus type testing. Among these three techniques ABO type testing and Rhesus type testing is most widely used in all laboratories. In ABO testing, the antigen is added to the blood sample and based on the reaction between antigen and antibody, diagnosis is made. In Rhesus type of testing, the positive and negative type of the blood group can be determined. Although, it is simple to detect but in handling with large number of samples, it is tedious to do and it may also leads to wrong analysis. To overcome these problems, the proposed LASER technique is used. This is based on the principle, that the LASER intensity changes due to the occurrence of clumping in the blood sample which in turn changes the density of the blood sample. This variation is sensed by the level of the energization of the photocell. The output from the detector is in the form of voltage which is fed to the comparator which decides the blood group using embedded controller.
\end{abstract}

Keywords: LASER beam, antibody, Photocell, Clumping, Antigens, Blood sample, Embedded. ****

\section{INTRODUCTION}

Determination of blood group of any human being is a vital factor. Man by nature has any one of the Blood group namely $\mathrm{A}, \mathrm{B}, \mathrm{AB}$ and $\mathrm{O}$. The blood group " $\mathrm{AB}$ " is called the "Universal acceptor" and the people with the "O" group are called "Universal donor". During blood transfusion any mismatch may even lead to the death of a person. Hence it is of utmost importance for every person to identify bloodtheir group.

In general, the technicians in laboratories are determining the blood group by using ABO blood testing method. This testing is under the basis of aggregation process of antigen and antibody. Here the antibody is a collection of toxins, bacteria, foreign blood cells and the cells of transplanted organ. In the way of manual analysis, we can able to get better results but inhandling with wide range of samples, the examining person experience very tough joband may misdeed to maintain the blood sample and records accurately. To avoid such a situation, here a new solution is proposed to recognize the blood group. In this new technique, the LASER source and photocell are used to analyse the $\mathrm{ABO}$ blood grouping $(\mathrm{A}, \mathrm{B}, \mathrm{O})$ and Rhesus type testing $(\mathrm{Rh}+\mathrm{ve}$ and-ve).

Presently, an average of 200 to 300 blood samples are analysed within 2 to 3 hours which can be carried out only by technicians but when the proposed device is used the same task can be completed within a short period of time with good precision. One more added advantage of this proposed device is of affordable cost.

\section{LITERATURE SURVEY}

T.M.Selvakumari ${ }^{[1]}$, Blood group detection using fibre optics.In this technique, the transmitter is used to generate pulses of frequency 10KHZ.Then these pulses are fed to the Light Emitting Diode [LED], which converts electrical variations into optical variations. After that the optical signals were launched into the fibre. Then it is fed to the blood sample and it is received by the receiver which converts the optical variations again into electrical variations. The observed electrical variations are different for all blood types.Due to the optical variations of different blood group,there will be corresponding voltage variation in the output of the photo detector. Thereby the blood groups (ABO) can be determined without using the antigen. But, the $\mathrm{Rh}$ (positive and negative) type of the blood group has not discussed.

S.M. NaziaFathima [2], Classification of blood type by microscopic colour images. In this semi-automated system, the blood group can be identified by microscopic colourimages. Initially it performs image pre-processing by histogram equalization and colour correction and then colour space conversion for converting the RBC to HIS. Then, it extracts the colour and texture feature of the images 
using cumulative histogram and haralick method respectively. Then finally the corresponding person's blood group can be analysed by using Support Vector Machine (SVM). In this system, the more skilled persons are needed to handle and it is tedious to do.

P.A.Berlitz ${ }^{[3]}$, Rapid Automated Blood Group Analysis with QCM Biosensors. In this type of analysis, protein ' $A$ ' coating is provided on the gold surface of QCM biosensors for the immobilization of antibodies against blood group antigens $\mathrm{A}$ and $\mathrm{B}$ which permits the identification of the blood groups with two measurements. But for determining $\mathrm{Rh}$ factor one more experiment is needed. Here the computer and software is essential to detect the blood group. It also requires chemicals and reagents for the determination and that is expensive.

Ferraz, Ana ${ }^{[4]}$, Automatic system for determining of blood type using image processing technique. In this system, the blood group can be determined using image processing technique with LABVIEW and IMAQ vision. Here the image of that blood sample is captured after the slide test is performed which detect the occurrence of agglutination reaction (clumping). Next the classification of algorithm is used to determine the blood grouping. Finally, all the information is stored in the database. This system is not fully automated because the slide test is conducted manually and it also consumes more time for image processing.

Brinkhues O, Giers G, Hanfland ${ }^{[5]}$, Electronic data processing-assisted serial automation of current methods in blood group serology. The introduction of special centrifugal racks with a transparent bottom into the conventional typing of blood group in glass tubes facilitates the simultaneous work on and reading of a maximum of 32 complete $\mathrm{ABO}$, Rhesus and Kell typing in one series. As a result of the facts that it is unnecessary to label the individual tubes and that the pipetting of serum and erythrocyte suspension is done automatically and through the unmistakable classification of the samples by means of bar-coding, the manual work is reduced to about 50\%. Even though it is useful but it is semi-automated system. So the working personnel have some difficulties.

K.Satoh, Y.Itoh ${ }^{[6]}$, Forensic ABO blood grouping by 4SNPs analyses using an ABI PRISM 3100 genetic analyser. This paper proposed a new method to classify the blood grouping by using PCR-based methods, such as sequence-specific primers with a positive control (PCR-SSPPC) and confronting two pairs of primers (PCR-CTPP) for forensic $\mathrm{ABO}$ groupings using fragment analysis by ABI PRISM 3100 genetic analyser. The method allows the wellestablished base changes at four nucleotide positions 261 , 796,802 and 803 to be assayed, so that reliable blood group estimation is established by the presence of three representative alleles such as A,B and $\mathrm{O}$. This process consumes more time to complete the entire task.

\section{PROPOSED METHOD}

Two techniques are widely used for blood group analysis. Among these the most widely used one is ABO technique. In this antigen $\mathrm{A}$, antigen $\mathrm{B}$ and antigen $\mathrm{D}$ is used for analysing the blood group. According to $\mathrm{ABO}$ and $\mathrm{Rh}$ blood grouping system, the person can belong to any one of the following eight blood groups: A positive, A Negative, B positive, B Negative, AB positive, AB Negative, $\mathrm{O}$ positive, O Negative.

While doing the blood group analysis by manual process in laboratories, a drop of antigen is added with a drop of blood sample and technician has to wait for few seconds, to check whether the clumping reaction has occurred or not. Based on the level of reaction occurred, individual's blood group can be identified by the technicians.

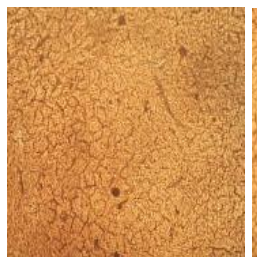

(a)

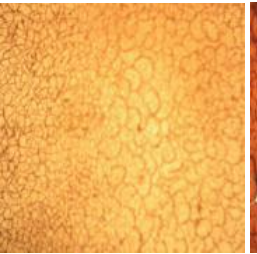

(b)

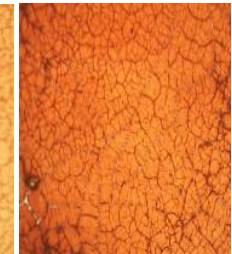

(c)
Fig-1: Agglutination reaction in $\mathrm{O}$-ve blood group

Fig. 1(a, b, c) shows the O Negative blood group sample on adding Antigen-A, Antigen-B and Antigen-D respectively. In this blood sample it was observed that no clumping has occurred with any one of the antigen ${ }^{[2]}$.

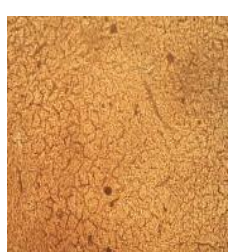

(a)

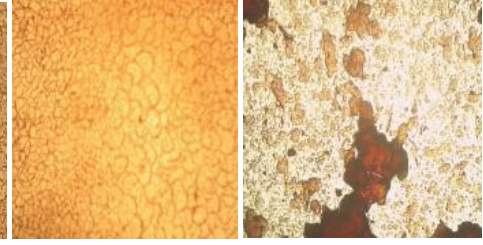

(b) (c)
Fig-2: Agglutination reaction in $\mathrm{O}+\mathrm{ve}$ blood group

Fig. 2(a, b, c) shows the O Positive blood group sample on adding Antigen-A, Antigen-B and Antigen-D respectively. In this blood sample it was observed that the clumping occurred only for the Antigen-D ( $\mathrm{Rh}$-positive and Negative) ${ }^{[2]}$.

In this conventional method, there are some drawbacks like less accuracy, more time consumption and it is tedious while handling with huge samples. To overcome these problems, proposed LASER technology is used and it reveals a very big change in medical field.

In this proposed technique, LASER input and photocell plays a major role. Basically photocell is a semiconductor device which absorbs light energy and converts it into electrical energy.Fig.3 shows the block diagram of Automation in Blood Group Analysis Using LASER Technology. Initially the LASER beam is allowed to pass to 
the photocell, then the photocell starts to energize and the voltage from the cell is measured as 2.5 volts.

Blood sample for which blood group is to be identified is placed in the glass slide between the LASER beam and photocell. After these arrangements, the voltage from the photocell is decreased from $2.5 \mathrm{Vdue}$ to the variations in LASER beam intensity. Then a drop of antigen is added to a drop of blood sample which is on the transparent glass slide.

If the clumping (the agglutination reaction) occurs, then the density of the blood sample changes (high) which in turn changes intensity of light to the photocell. So automatically the level of energization of the photocell is decreased. If the clumping reaction does not occur, then the density of blood sample does not change. So there will be no changes in the level of energization of photocell.

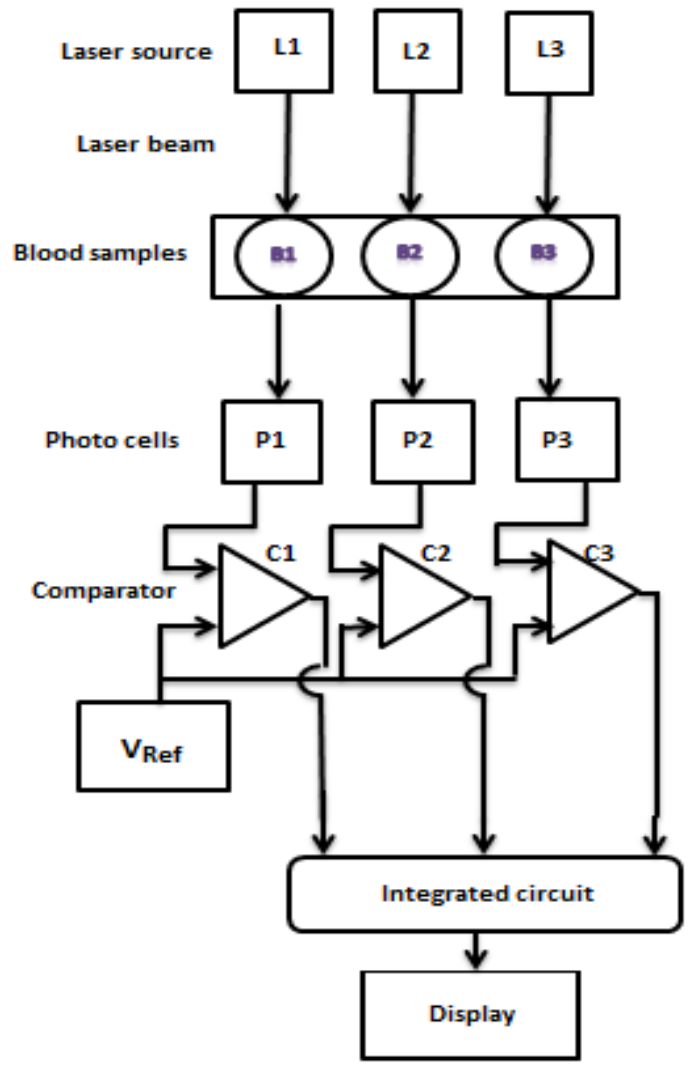

Fig-3: Block diagram of proposed system.

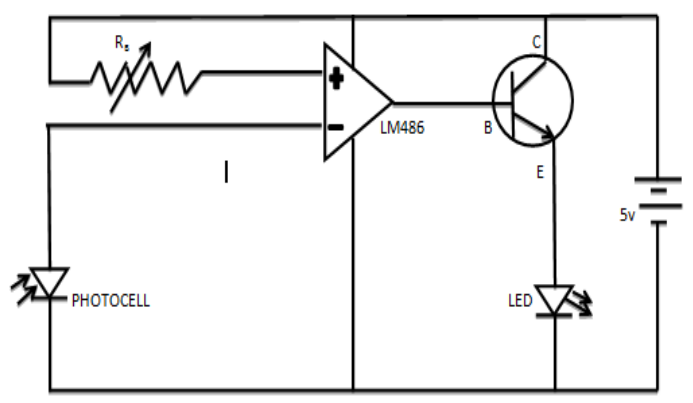

Fig-4: Circuit Diagram for Binary Output.
Then the output from the photocell is fed to the comparator which is used to compare these voltages with reference voltage. Then the error signal is given as the input to the NPN Transistor. If the difference between the two voltages is positive, then the transistor conducts then there will be a current flow in the circuit. Now the binary output is ' 1 '. Otherwise the transistor does not conduct because the Vcc supply to the emitter junction is also negative. So there is no current flows in that circuit, then the binary output is ' 0 '. It is again fed to Microcontroller for finding the individual's blood group. The above process needs to do with three antigens for analysing the blood group. The antigens are Anti-A for 'A group', anti-B for 'B group' and anti-D for ' $\mathrm{Rh}$ factor'. The combination of binary codes for identifying different types of blood group is given in table 1

Table1: binary output and its corresponding blood group.

\begin{tabular}{|l|l|l|l|}
\hline Anti A & Anti B & Anti D & $\begin{array}{l}\text { Blood group of } \\
\text { individuals }\end{array}$ \\
\hline 1 & 0 & 1 & A positive \\
\hline 1 & 0 & 0 & A negative \\
\hline 0 & 1 & 1 & B positive \\
\hline 0 & 1 & 0 & B Negative \\
\hline 1 & 1 & 1 & AB Positive \\
\hline 1 & 1 & 0 & AB Negative \\
\hline 0 & 0 & 1 & O positive \\
\hline 0 & 0 & 0 & O Negative \\
\hline
\end{tabular}

In this proposed automation technique, instead of using the combination of LASER and photocell, the combinations of Light Emitting Diode (LED) and Light Dependent Resistor (LDR) can also be used for analysis of ABO blood typing and Rhesus $(\mathrm{Rh})$ type. Here the output light intensity of the LED changes due to the density of the blood sample so that the resistivity of the LDR also changes which changes the output voltage. From the voltage variation, the blood group can be identified.

\section{EXPERIMENTAL SETUP}

The simplest experiment was made for the analysis of person's blood group using LASER beam and photocell. In this proposed technique, the individual's blood group can be decided on the basis of variations in the photocell energization due to the variation in the intensity of the LASER beam. Initially particular intensity of the LASER beam is taken, and then it is placed at the height of $21 \mathrm{~cm}$ above the photocell. Now the photocell is energized due to the absorption of light energy and the voltage is noted as 2.5V.Then the glass slide with blood sample is placed between LASER and photocell to the analysis of person's blood group. Here the voltage is decreased to 2.35 volts. Then the drop of anti-D is added in that blood sample to determine Rhesus type (positive and negative).

Because of antigen and antibody reaction in the blood, the clumping (agglutination reaction) is occurred, so that the 
blood is said to be as positive type. Then the density of the blood sample is increased by clumping reaction that has occurred in that sample. So that the voltage from the photocell decreased to $2.0 \mathrm{~V}$ due to the variations in the intensity of the LASER beam

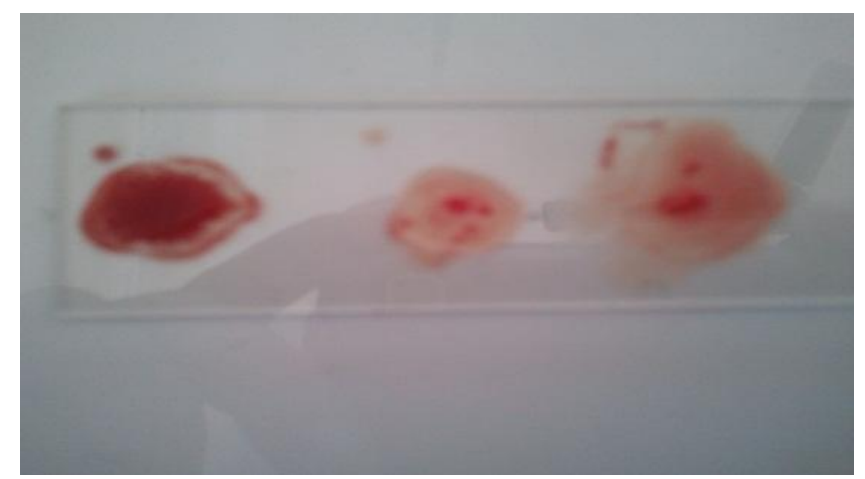

Fig-5: Clumpingin O-Positive blood group

After this, the same experiment was made with negative type blood sample, and it was observed that no clumping reaction occurs. So no changes takes place the density of blood sample and the voltage obtained from photo cell is same as that of 2.3 volts. From these, voltage variations the blood group has been analysed.

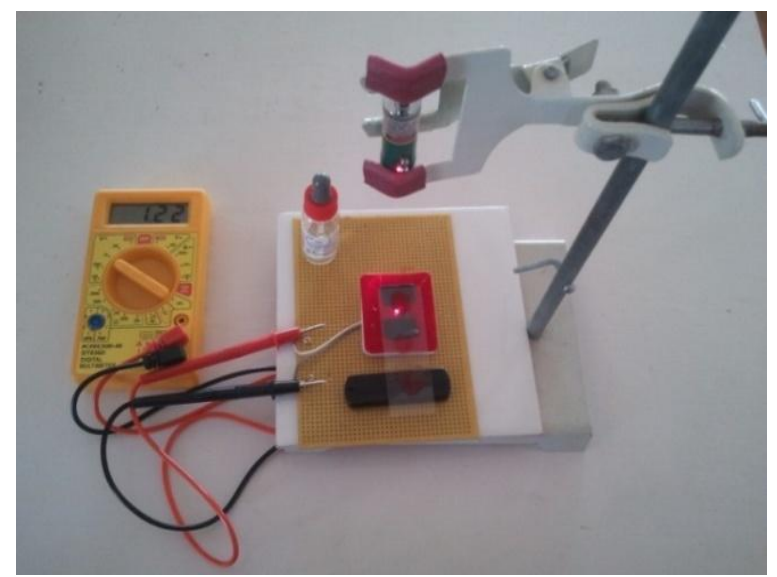

Fig- 6: Experimental setup for blood group analysis.

\section{CONCLUSIONS}

Generally, the blood group analysis can be done only by manual process. Nowadays, Automation plays a prominence role in modern technology development. This paper has proposed an automation method of blood group identification using LASER technology. The corresponding individual's blood group can be decided by using the variations in the level of photocell energization due to the changes in the LASER beam intensity. This automation device has appreciable advantages like smaller in size, lower in cost, no side effects, better accuracy, less time consumption and then it overcomes the present difficulties of manual process. If the proposed methodology will be implemented in a real time work, it can able to make great change in a medical field.

\section{REFERENCES}

[1]. Selvakumari, T.M. (2011).Blood GroupDetection Using Fibre optics, American Journal of Physics, vol.4, issue 3, pp.165-168.

[2]. NaziaFathima.S.M. (2013).Classification of blood types by microscopic colour images, International journal of machine learning and computing.vol 3, no. 4, pp.376-379.

[3]. Berlitz,P.A., claussen, J., Doring, J.S., Drechsel, H., Northoff, H., andGehring,F.K.(2012).Rapid Automated blood group analysis with QCM Biosensors, $5^{\text {th }}$ European Conference of International Federation for Medical and Biological Engineering IFMBE proceedings, volume 37,pp 1039-1042.

[4]. Ferraz, Ana, Member, IEEE (2013). Automatic system for determining of blood type using image processingtechnique. Bioengineering (ENBENG), IEEE Feb $20-23$.

[5]. Brinkhues, O., Gries, G., Hanfland, P., (2003).Electronic data processing- assisted serial automation of current methods in blood group serology, ThrombRes, 108:195-96

[6]. Satoh, K., Itoh, Y.(2006).forensicABO bloodgrouping by 4 SNPs analyses using an ABIPRISM 3100 genetic analyser, International Congress Series 1288 (2006) 49-51.

[7]. Fiji, Hosoi, (July.2008).Biological and clinical aspects of ABO blood group system vol.55, pp.770-8509.

[8]. Hearn Shaw, k. (Nov 2004).Minimizing the risk associated with having a blood transfusion.

[9]. Ryszard, S., Romaniuk, and Jerzy Gajda (2013).LASER Technology and Application 2012, International Journal of Electronics and telecommunications, vol.59, pp.195-202.

\section{BIOGRAPHIES}

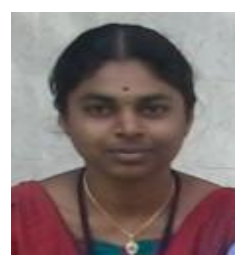

PRIYADHARSHINI.R is pursuing Bachelor of Engineering in the discipline of Electrical and Electronics Engineering at Knowledge Institute of Technology, Salem, under Anna University, Chennai, India. She has presented number of technical papers in symposium. She is the acting student member of various cells and forums like Robotics Intelligent Club, Green club, I-can Club, Instrumentation and Engineers club. She won prizes for project contest. Presently she is doing minor research works on various fields like space science, embedded systems Biomedical Instrumentation and Robotics etc. She is highly appreciated by the Head of the Department.

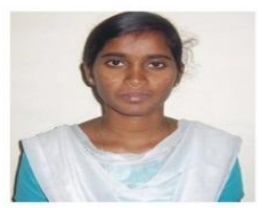

RAMYA.S is pursuing Bachelor of Engineering in the discipline of Electrical and Electronics Engineering at Knowledge Institute of Technology, Salem, under Anna University, Chennai, India. She has presented number of technical papers in symposium. She is the acting student member of various cells and forums like Robotics Intelligent Club, Green club and Science club. She won prizes for project contest. Presently she is doing minor research works on various fields like space science, 
embedded systems Biomedical Instrumentation and Robotics etc. She is highly appreciated by the Head of the Department.

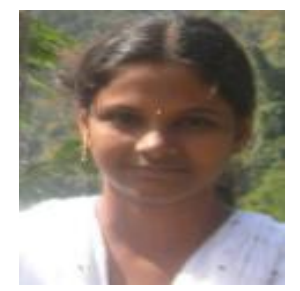

KALAIYARASI.S is pursuing Bachelor of Engineering in the discipline of Electrical and Electronics Engineering at Knowledge Institute of Technology, Salem, under Anna University, Chennai, India. She has presented number of technical papers in symposium. She is the acting student member of various cells and forums like Robotics Intelligent Club, Green club, I-can Club and Science club. She won prizes for project contest. Her performance towards academic is excellent and she was awarded as the academic topper of the class for the past three semesters in the department. Presently she is doing minor research works on various fields like space science, embedded systems Biomedical Instrumentation and Robotics etc. She is highly appreciated by the Head of the Department.

S.KALPANADEVI is currently working as an Associate Professor in the Department of Electrical and Electronics Engineering at Knowledge Institute of Technology, Salem. She received his UG degree in the discipline of Electrical and Electronics Engineering from Kongu Engineering college under Bharathiyar University, Coimbatore and got PG degree in Process Control and Instrumentation discipline from Annamalai University, Chidambaram. She has published and presented number of technical papers in National and International Journals and Conferences. She is a life member of ISTE. She has guided number of projects for students. She has organized many guest lectures in various fields. Her research interests lie in the field of Control System, Medical Instrumentation and Embedded System.

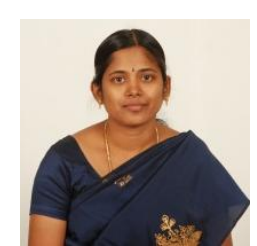

Dr.N.SUTHANTHIRA VANITHA is currently working as a Professor and Head of the EEE Department at Knowledge Institute of Technology, Salem. She received the B.E. - Electrical and Electronics Engineering from K.S.R. college of Tech, Tiruchengode in 2000 from Madras University, M.E. - Applied Electronics in Mohamed Sathak Engineering College in 2002 from Madurai Kamaraj University and Ph.D., in Biomedical Instrumentation \& Embedded Systems in 2009 from Anna University, Chennai. She is life member of ISTE \& CSI. Her research interests lie in the area of Robotics, DSP, MEMS and Biomedical, Embedded Systems, Power Electronics and Renewable Energy systems, etc. She has published and presented number of technical papers in National and International Journals and Conferences. She has guided number of Projects for UG and PG students, currently guiding $12 \mathrm{Ph} . \mathrm{D}$., scholars. 